\title{
Muscular dystrophy in PTFR/cavin-1 null mice
}

\author{
Shi-Ying Ding, ' Libin Liu, ${ }^{1}$ and Paul F. Pilch, \\ ${ }^{1}$ Department of Biochemistry, ${ }^{2}$ Department of Medicine, Boston University School of Medicine, Boston, \\ Massachusetts, USA.
}

Mice and humans lacking the caveolae component polymerase I transcription release factor (PTRF, also known as cavin-1) exhibit lipo- and muscular dystrophy. Here we describe the molecular features underlying the muscle phenotype for PTRF/cavin-1 null mice. These animals had a decreased ability to exercise, and exhibited muscle hypertrophy with increased muscle fiber size and muscle mass due, in part, to constitutive activation of the Akt pathway. Their muscles were fibrotic and exhibited impaired membrane integrity accompanied by an apparent compensatory activation of the dystrophin-glycoprotein complex along with elevated expression of proteins involved in muscle repair function. Ptrf deletion also caused decreased mitochondrial function, oxygen consumption, and altered myofiber composition. Thus, in addition to compromised adipocyte-related physiology, the absence of PTRF/cavin-1 in mice caused a unique form of muscular dystrophy with a phenotype similar or identical to that seen in humans lacking this protein. Further understanding of this muscular dystrophy model will provide information relevant to the human situation and guidance for potential therapies.

Conflict of interest: The authors have declared that no conflict of interest exists.

Submitted: October 3, 2016

Accepted: January 24, 2017

Published: March 9, 2017

Reference information: JCI Insight. 2017;2(5):e91023. https:// doi.org/10.1172/jci.insight.91023.

\section{Introduction}

Muscular dystrophies (MDs) are a group of diseases of variable severity that are caused by mutations in a relatively large number of different genes, which cause progressive loss of muscle function (1). Most of the mutated genes causing MDs are involved in sarcolemmal functions, the maintenance of membrane integrity, and/or communication with the extracellular matrix (2). Two such MD genes, caveolin-3 $(C A V 3)(3,4)$ and polymerase I transcription release factor $(P T R F)(5-8)$, encode protein components of cell surface domains called caveolae in humans and mice (Cav3 and Ptrf).

Caveolae are small flask-shaped invaginations of the plasma membrane that play important roles in a number of cellular processes, and the most recent evidence documents their role in organizing and stabilizing the plasma membrane in many different cell types including skeletal muscle cells (9-12). Proteins required for caveolae formation include caveolin-1 (Cav1) for nonmuscle tissues (most abundant in adipocytes, endothelium, and lung), Cav3 for skeletal and cardiac muscle, and PTRF/cavin-1 for caveolae in all cells/tissues. Additional members of the cavin family, cavins-2-4, appear to influence caveolae structure in a more restricted fashion (12). Caveolae are abundant in the sarcolemma of skeletal muscle where they play important roles in muscle physiology, as is best documented by evidence from rodent models of caveolae deficiency and from the phenotype(s) of human caveolopathies $(4,13)$.

Cav3 null mice have mild-to-moderate myopathic changes, including variability in muscle fiber size and the presence of necrotic fibers (14), as well as muscle degeneration (15). In humans, mutations in the CAV3 gene cause a number of different muscle pathologies depending on the mutation, including autosomal dominant limb-girdle muscular dystrophy 1C (LGMD1C), rippling muscle disease, hyperCKemia, and myalgia (16). More recently, human mutations in PTRF have been reported and they result in congenital generalized lipodystrophy type 4 (CGL4), a rare autosomal recessive disorder characterized by near total absence of body fat $(5,7,8,17-20)$. While CGL4 is the defining pathology of these patients, they also exhibit MD and cardiac abnormalities including arrhythmias that can be fatal.

The involvement of caveolae components in human muscle disease emphasizes their importance in muscle function, both mechanically and metabolically. We have described aspects of the metabolic phenotype for PTRF/cavin-1 global KO mice, the equivalent to humans with null mutations in this gene. Like humans, these mice have a markedly reduced fat mass, dyslipidemia, insulin resistance, and glucose 
intolerance $(21,22)$. Lo et al. recently described altered skeletal muscle morphology in PTRF/cavin-1 null mice and in zebra fish having a muscle-specific cavin-1 knockdown (23). They showed an essential mechanoprotective role of caveolae in maintaining sarcolemmal integrity in skeletal muscles under conditions of enhanced membrane tension. In the present study, we investigated the effects of cavin-1 deficiency on skeletal muscle biochemistry and physiology, including muscle growth, mitochondria function, and myofiber composition. Our findings show that PTRF/cavin-1 null mice develop a muscular dystrophic phenotype similar to the clinical features observed in human patients with PTRF mutations. Therefore, these animals represent a valuable tool for understanding caveolae function in skeletal muscle at a molecular level and for developing potential therapeutic approaches for human patients.

\section{Results}

Cavin-1 null mice develop muscle hypertrophy. Mice lacking cavin-1 show muscle hypertrophy, as shown for the entire hindlimb muscle in Figure 1A. The total body weight of cavin-1 KO mice was not significantly different than that of WT animals despite the significant reduction in fat mass (see also refs. 21, 22). Thus, we measured muscle weights in isolated tibialis anterior (TA), extensor digitorum longus (EDL), soleus, gastrocnemius and plantaris $(\mathrm{G} / \mathrm{P})$, and heart, and the mass of all muscles except the TA was significantly increased in cavin-1 KO mice as compared with WT (Figure 1B). This difference was even more pronounced with age when the total body weight of the $\mathrm{KO}$ mice is approximately $20 \%$ less than that of WT (Supplemental Figure 1; supplemental material available online with this article; https:// doi.org/10.1172/jci.insight.91023DS1). Morphometric and histological examination revealed increased fiber size, quantified as mean cross-sectional area (CSA), that was $25 \%$ higher in KO mice as compared with WT mice (Figure 1, C and D). Centrally nucleated muscle fibers were quantified in soleus from WT and $\mathrm{KO}$ mice. Of the total muscle fibers, $10.5 \%$ in cavin- $1 \mathrm{KO}$ mice were centrally nucleated compared with $0.4 \%$ in WT mice (Figure 1E), and this phenomenon is characteristic of muscle regeneration in response to disease or injury. $\mathrm{MyoD}$ is the master regulator of myogenesis and muscle development (24) and its expression was the same in WT and cavin-1 KO mice (Figure 1F), indicating that the lack of caveolae does not affect this process. As expected from the previously reported results of whole-muscle analyses (22), loss of cavin-1 resulted in pronounced loss of Cav1 and Cav3 proteins in all of the muscle types we tested (Figure 1F). We also examined the mRNA expression of genes encoding several myogenic markers. Expression of Myog (which encodes myogenin) and Myf6 was slightly upregulated in soleus (Supplemental Figure 2A), but not in G/P muscles of KO mice (Supplemental Figure 2B). There was no change in mRNA levels of other myogenic markers, such as Pax7 and Myf5, which is consistent with the normal MyoD expression in KO mice. In addition, in WT mice, among TA, EDL, soleus, and G/P muscles, the highest cavin-1 protein expression was observed in soleus muscle (Figure 1F and Supplemental Figure 3), which may explain the most marked morphological change indicated by the largest number of centrally nucleated myofibers in soleus muscle of cavin-1 KO mice.

Muscle hypertrophy in cavin-1 null mice is associated with constitutive Akt activation. Muscle growth and the maintenance of skeletal muscle structure are positively regulated by the IGF-1/Akt signaling pathway. Activation of the Akt pathway by IGF-1 results in increased protein synthesis and hypertrophy via mTOR (25-27). We therefore compared expression of a number of relevant species in WT mice and their null littermates, and as shown in Figure 2A, the basal phosphorylation state of Akt, and other Akt signaling pathway downstream factors such as p70S6k, 4EBP1, and S6 were all increased in the cavin-1 KO mice as compared with WT, indicative of constitutive activation of Akt. Increased phosphorylation of p70S6k, S6, and $4 \mathrm{EBP} 1$ was consistent with activation of mTOR, which results in increased protein synthesis.

We also measured the downstream response to IGF-1 administration in vivo in paired littermates as shown in Figure 2B and found that Akt and other downstream mediators of IGF-1 all showed enhanced phosphorylation due to the growth factor in WT mice. In KO mice, the response to IGF-1 was minimal, despite the basal phosphorylation state of Akt and the other targets being as high or higher in the cavin-1 null animals as in the stimulated state in WT. Quantification of the Western blots showed that with IGF-1 stimulation, the ratios of p-p70S6K to total p70S6K increased $69 \%$ in WT muscle and $10 \%$ in KO muscle compared with the ratios at basal status $(P<0.001)$. Changes in $\mathrm{p}-\mathrm{S} 6 /$ total S6, $\mathrm{p}-4 \mathrm{EBP} 1 /$ total $4 \mathrm{EBP} 1$ and p-Akt/total Akt all suggested relatively lower response to IGF-1 in KO mice. To confirm that the Akt pathway was constitutively activated, we immunoprecipitated Akt from muscle lysates of KO and WT mice and confirmed that the in vitro kinase activity towards a glycogen synthase kinase-derived (GSK-derived) 
A

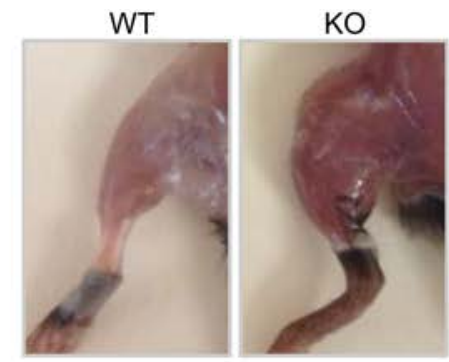

C
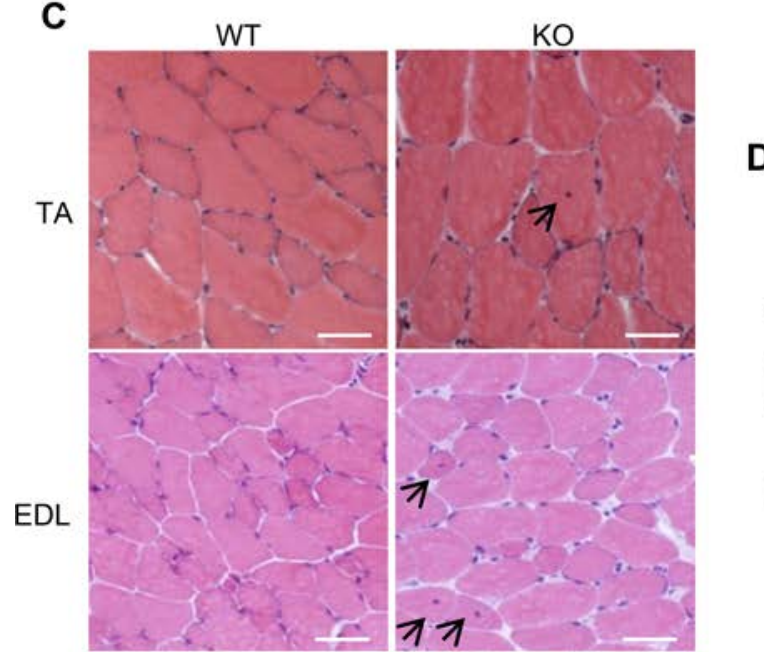

\section{$\pi \pi$}

B

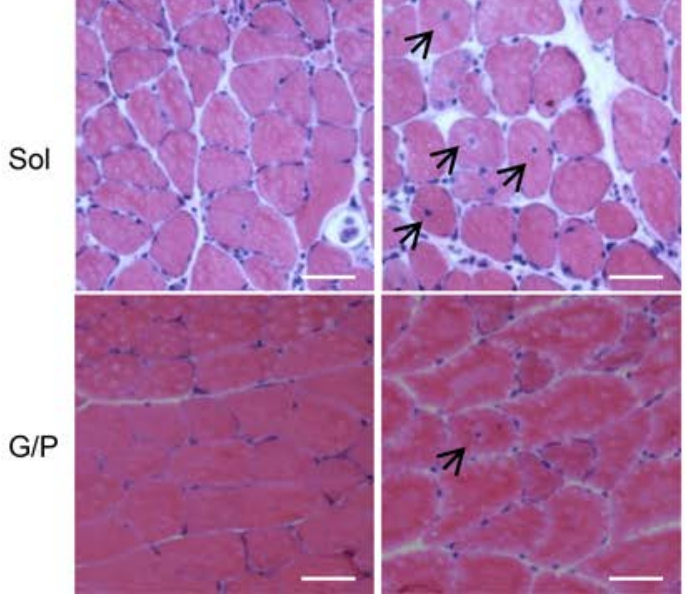

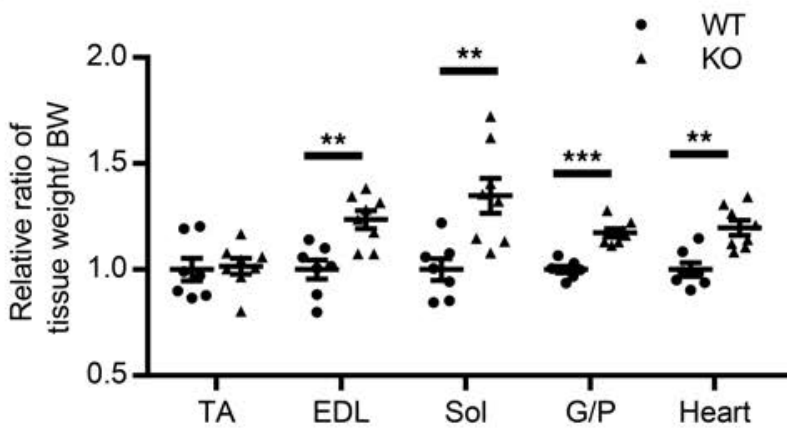

D

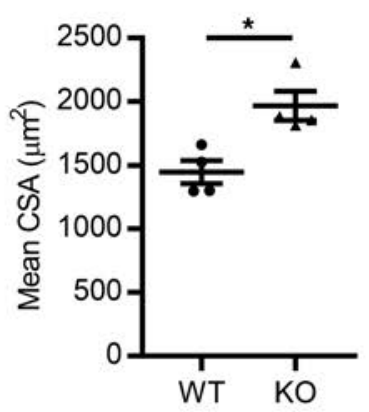

E

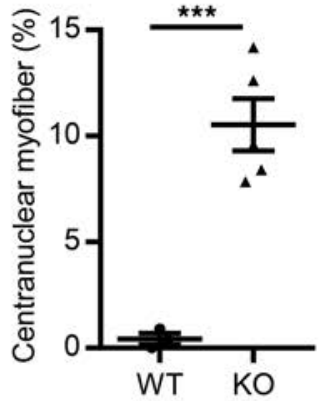

F
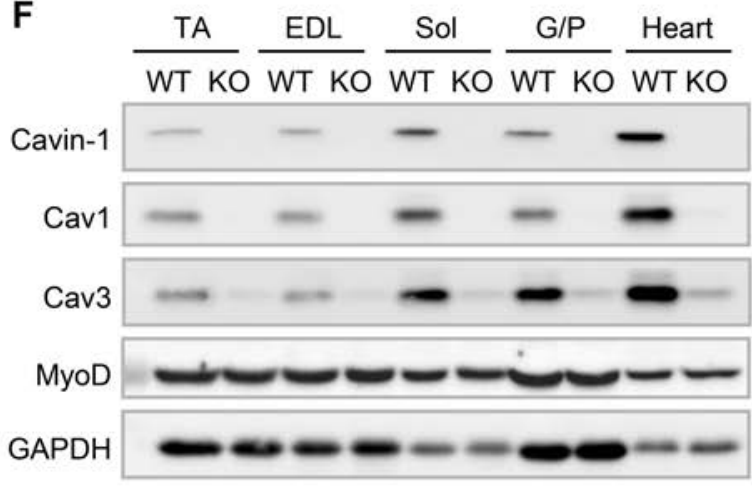

Figure 1. Deficiency of cavin-1 results in muscle hypertrophy. (A) Representative pictures of hindlimbs from 12-week-old WT and cavin-1 KO mice. (B) Relative ratio of muscle mass to body weight in WT and KO mice $(n=7-8)$. (C) Representative H\&E staining of tibialis anterior (TA), extensor digitorum longus (EDL), soleus (Sol), and gastrocnemius/plantaris (G/P) muscles of WT and KO mice at 12 weeks of age. Centralized nuclei are indicated with black arrowheads. Scale bars: $50 \mu \mathrm{m}$. (D) Analysis of the mean fiber cross-sectional area (CSA) in G/P muscles from 12-week-old WT and KO mice ( $n=4$, CSA from > 200 myofibers were measured for each mouse). (E) Percentage of centronuclear myofibers in Sol muscles of WT and KO mice at 12 weeks of age $(n=3-5)$. (F) Immunoblot analysis of caveolae proteins in TA, EDL, Sol, and G/P muscles, and hearts of WT and KO mice (representative of $n=3$ ). Cav1 and Cav3, caveolin-1 and -3 . Quantitative analyses are shown in Supplemental Figure 3. Data are represented as mean \pm SEM. Two-tailed Student's $t$ test was used for comparison between WT and KO mice. ${ }^{*} P<0.05,{ }^{* *} P<0.01,{ }^{* * *} P<0.001$.

peptide was indeed elevated when the kinase was obtained from the cavin-1 null animal (Figure 2C). The blots in Figure 2C also indicate increased basal phosphorylation of Akt and GSK in whole lysates from the same muscle samples, consistently suggesting an activated Akt pathway. Quantitative real-time PCR (q-PCR) analysis showed that the mRNA level of IgfI was upregulated in cavin-1 KO mice, while Mstn, which encodes myostatin, a negative regulator of muscle growth $(26,28)$, was diminished in the cavin-1 null muscles, consistent with its role in inhibiting muscle hypertrophy. We also examined 2 muscle atrophy genes which encode Atrogin-1 and MuRF-1 (29) and they were not significantly altered (Figure 2D). 
A

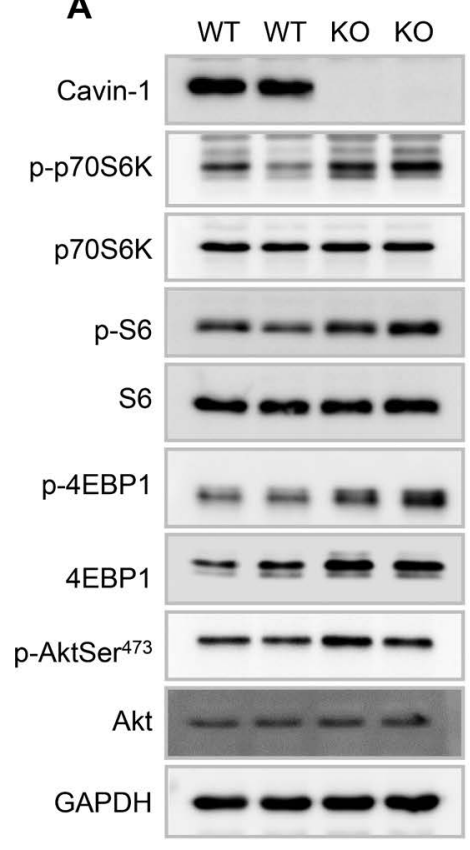

C

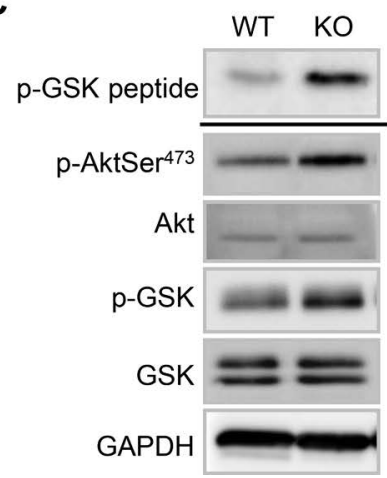

B
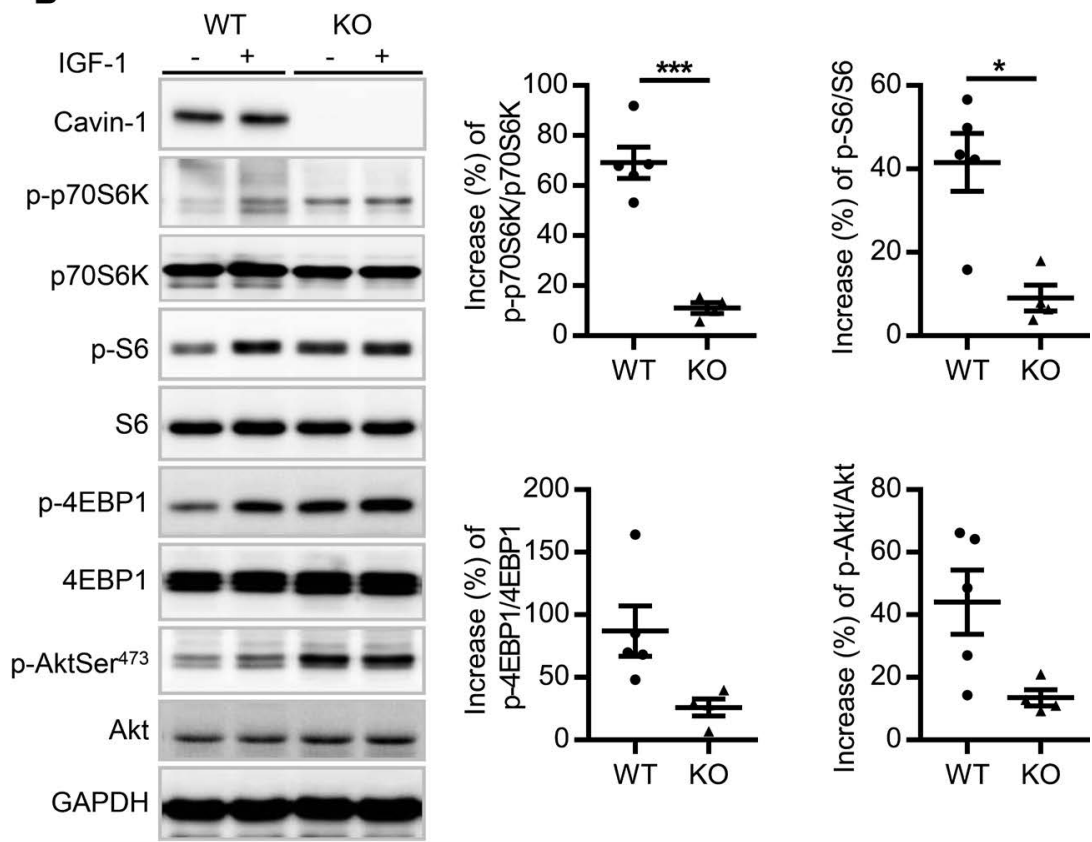

D
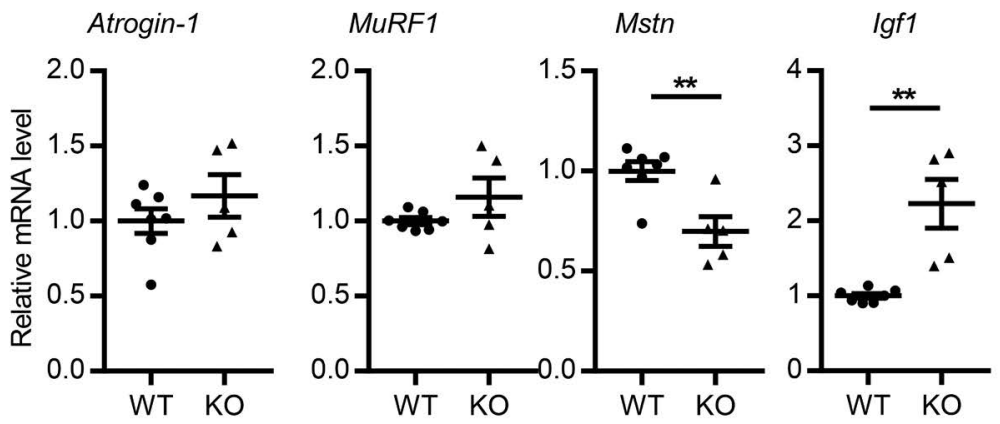

Figure 2. Cavin-1 null mice display constitutively enhanced Akt signaling. (A) Representative Western blot analysis of IGF-1/Akt signaling downstream factors in WT and cavin-1 KO littermates. The experiment was replicated 3 times (6 WT and 6 KO mice). (B) Representative Western blot analysis of IGF1/Akt signaling downstream factors with IGF-1 stimulation. The graphs show densitometric quantification of Western blots, representing increases (\%) of ratios of p-p70S6K/p70S6K, p-S6/S6, p-4EBP1/4EBP1and p-Akt/Akt, under IGF-1 stimulation compared with the ratios at basal status, respectively ( $n=4-5$ for each group from 3 independent experiments). (C) Representative Akt kinase assay from WT and KO tibialis anterior muscle. Akt was immunoprecipitated from muscle lysates and incubated with substrate and the kinase activity was measured using anti-phospho-CSK3 $\alpha / \beta$ to detect phosphorylation of glycogen synthase kinase (CSK) peptide (Akt substrate, $27 \mathrm{kD}$ ). The protein levels of total Akt, p-Akt, total GSK, and p-CSK in whole lysates of the same muscle samples were also detected in parallel in separate gels. The experiment was replicated 3 times. (D) mRNA expression levels of atrophy marker genes Atrogin-1 and MuRF1, and of important muscle growth regulator genes myostatin (Mstn) and Igf1 as measured by quantitative real-time PCR $(n=5-7)$. Data are represented as mean \pm SEM. Bonferroni's multiple comparison test (B) or 2-tailed Student's $t$ test (D) were used to determine the statistical significance between WT and KO mice. ${ }^{*} P<0.05,{ }^{*} P<0.01,{ }^{* * *} P<0.001$.

Cavin-1 null muscles display abnormalities in membrane integrity and muscle repair. The most common forms of $\mathrm{MD}$ result from mutations in the dystrophin-glycoprotein complex (DGC) and result in disruption of links between the extracellular matrix and the actin cytoskeleton and compromised sarcolemmal integrity $(30,31)$. We examined the protein contents of DGC components in WT and KO mice, and unlike the downregulation of DGC members seen in common MDs such as Duchenne muscular dystrophy (DMD) $(32,33)$ or the normal expression of these proteins observed in Cav3 null muscle (14), cavin-1 KO mice showed markedly elevated DGC protein levels, namely $\beta$-dystroglycan and $\alpha$-sarcoglycan (Figure 3A). Similarly, the cytoskeletal proteins $\alpha$-actin, $\beta$-tubulin, and vimentin were all dramatically upregulated, as were the membrane repair proteins MG53 (34) and dysferlin (35). Lastly, the proteins involved in muscle excitation-contraction coupling, such as dihydropyridine receptor (DHPR 2 2), a marker of the T-tubules, and ryanodine receptor (RyR1), a 
A

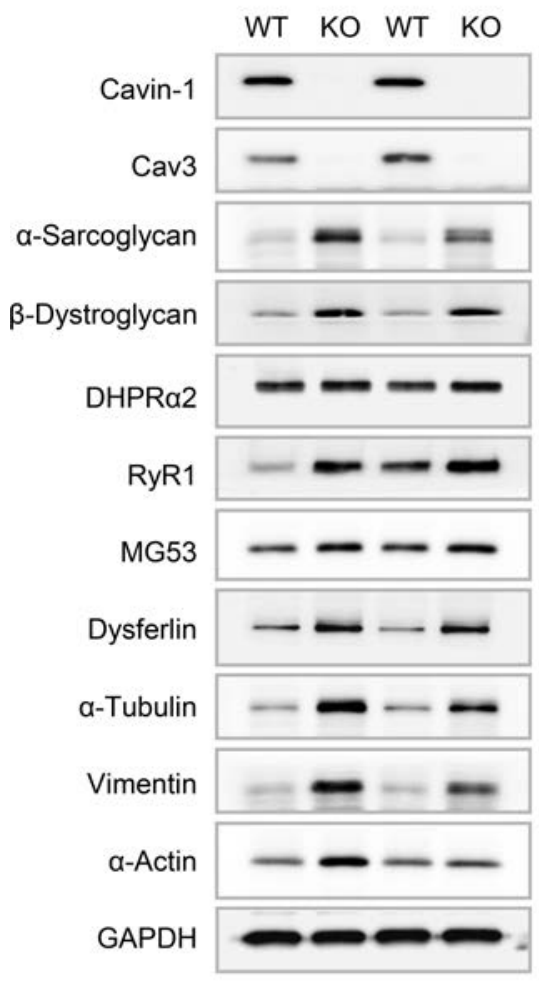

C

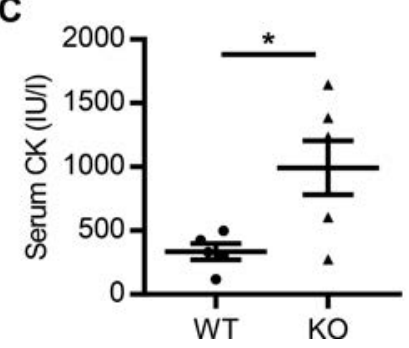

B
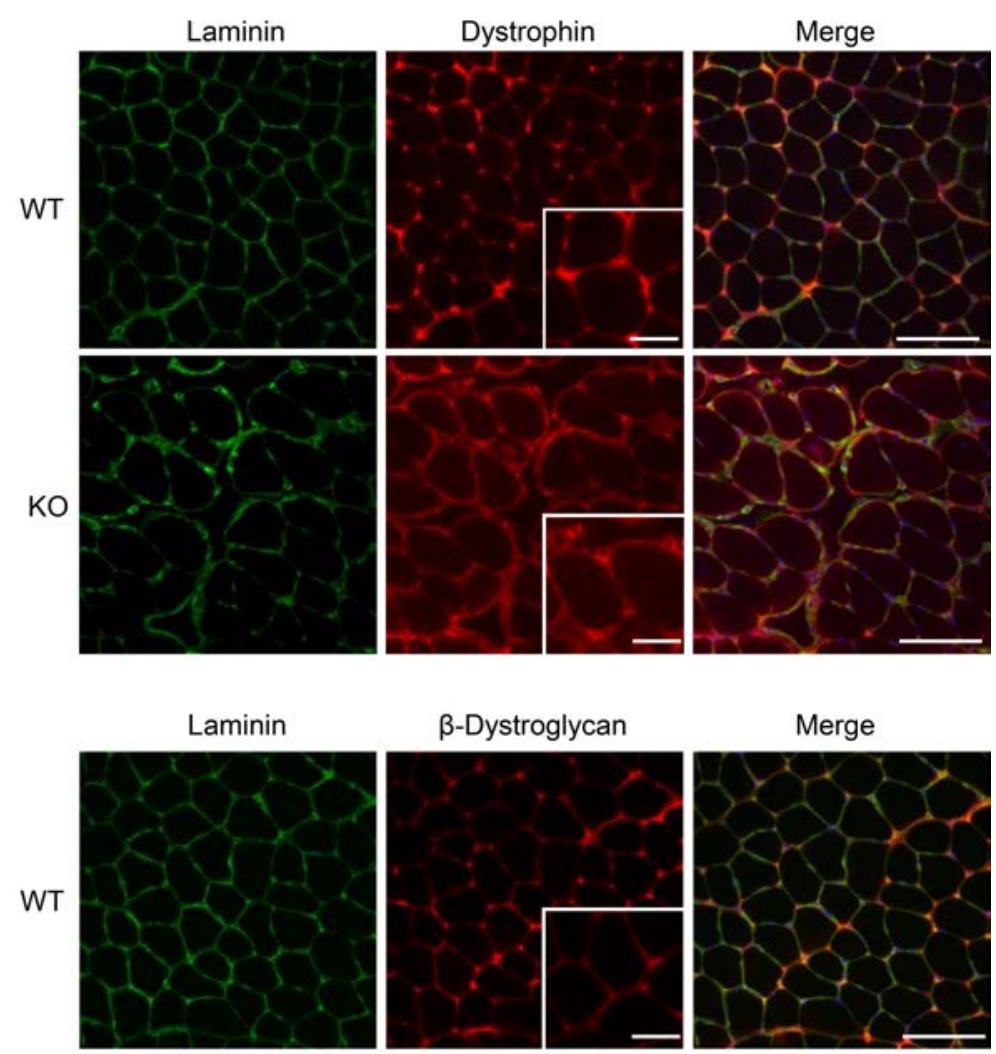

B-Dystroglycan
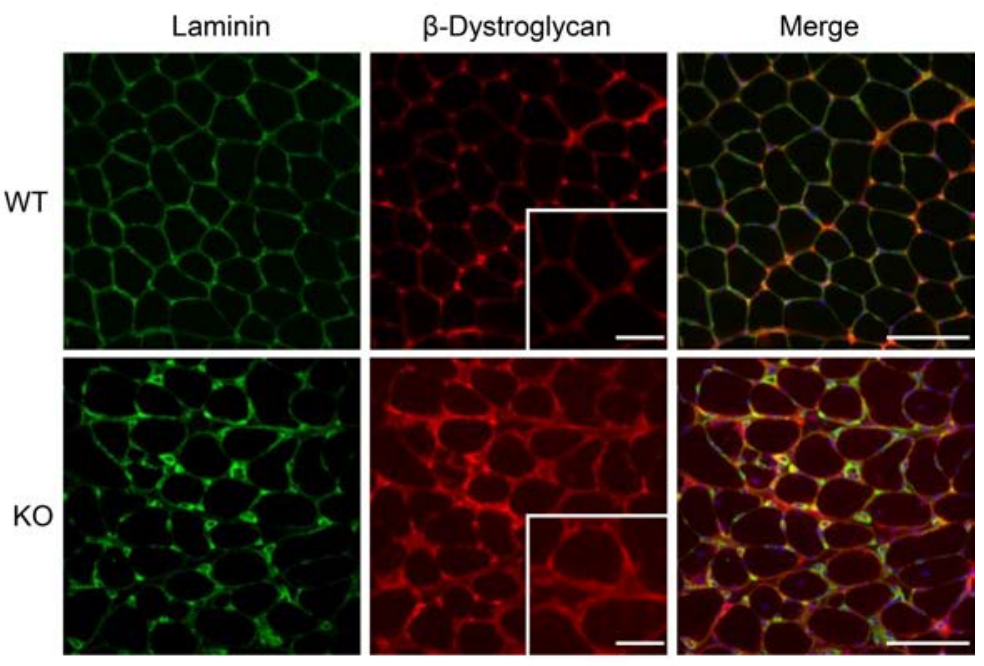

Figure 3. Cavin-1 null mice display elevated levels of dystrophin-glycoprotein complex (DGC) components, membrane repair proteins, and T-tubule-system components, accompanied with impaired sarcolemmal protein distribution and membrane integrity. (A) Representative Western blot analysis of muscular dystrophy-related proteins. Muscle extracts from 12-week-old WT and KO mice were immunoblotted with the indicated antibodies. Cav3, caveolin-3; $\alpha$-Sarcoglycan and $\beta$-dystroglycan are components of the DCC; dihydropyridine receptor (DHPR $\alpha 2$ ) is a marker of T-tubules; ryanodine receptor (RyR1) is a marker of the junctional sarcoplasmic reticulum; MG53 and dysferlin are membrane repair proteins; $\alpha$-tubulin, vimentin and $\alpha$-actin are involved in the cytoskeleton network. The experiment was replicated 3 times. (B) Dystrophin and $\beta$-dystroglycan immunofluorescence histochemistry (dystrophin and $\beta$-dystroglycan, red; laminin, green) on 8- $\mu \mathrm{m}$ cryosections of soleus muscle from 12-week-old WT and cavin-1 KO mice. Nuclei were stained with DAPI. Insets show increased intracellular immunofluorescence of dystrophin and $\beta$-dystroglycan in KO mice. Scale bars: $100 \mu \mathrm{m}$. (C) Serum creatine kinase (CK) levels in WT and cavin-1 KO mice ( $n=$ 5-6). Data are represented as mean \pm SEM. Two-tailed Student's $t$ test was used for comparison between WT and KO mice. ${ }^{*} P<0.05$.

marker of the junctional sarcoplasmic reticulum, were also increased in the KO mice (Figure 3A). To examine whether cavin-1 depletion affected the localization of DGC members, immunofluorescence analyses were performed. The data showed that dystrophin and $\beta$-dystroglycan were still present along the sarcolemma of fibers from $\mathrm{KO}$ mice, and their expression was markedly higher, as might be expected from the protein amounts (Figure 3B). In addition, very mild intracellular accumulation of these proteins was observed in KO myofibers, indicated by a slight but significant increase of intracellular fluorescence intensity (Supplemental Figure 4). Serum creatine kinase (CK) levels are an additional indication of muscle injury and membrane integrity and elevated serum CK levels are used clinically to diagnose MD (36). We observed 3-fold elevated serum CK levels in KO mice (Figure 3C), also consistent with impaired sarcolemmal integrity.

Cavin-1 null muscles are fibrotic and show impaired regeneration. We previously reported that cavin-1 deficiency in mice caused fibrosis in the caveolae-rich organs, such as fat and lung $(21,37)$. In skeletal muscle, fibrosis is a major pathological hallmark of chronic myopathies in which myofibers undergo successive 
A

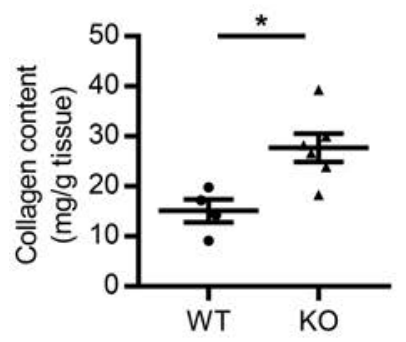

B

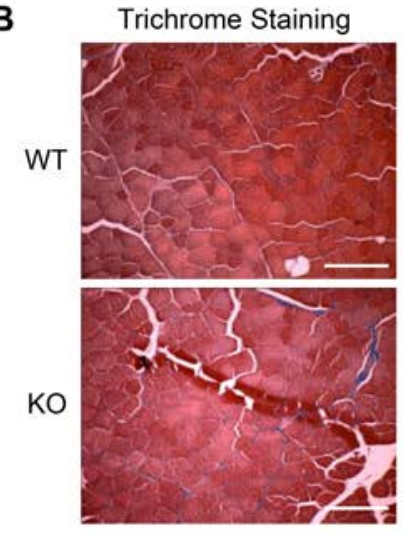

C

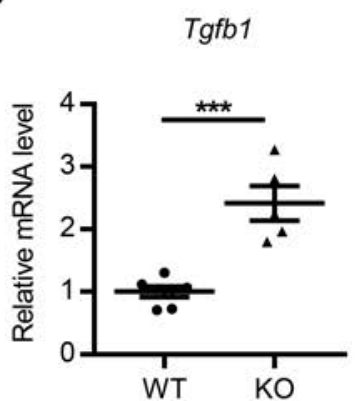

D

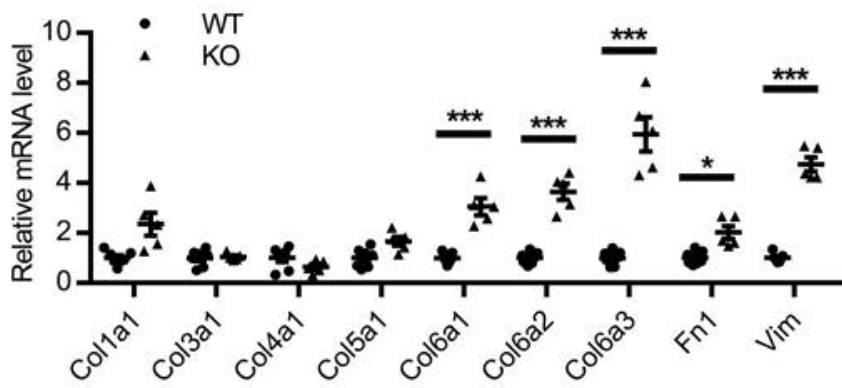

E

WT

KO

D3
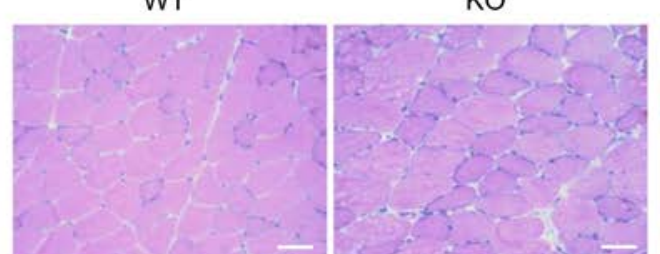

D7
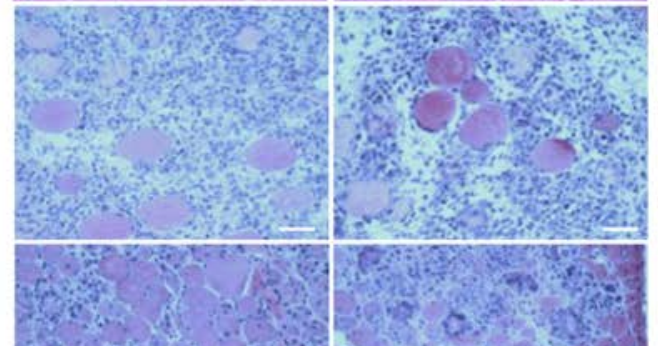

D14
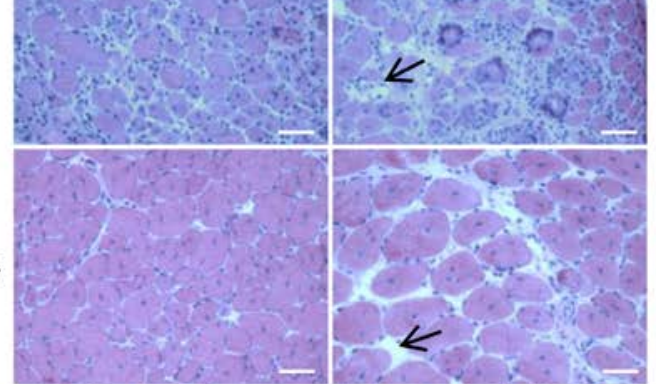

RO staining

(D14)
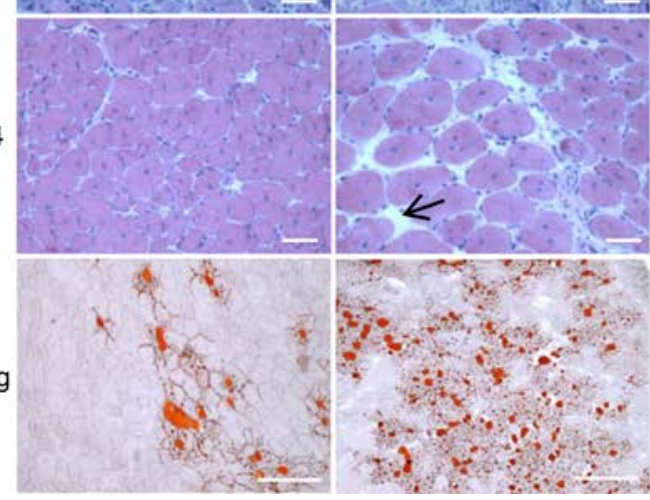

Figure 4. Cavin-1 KO mice show muscle fibrosis and impaired myofiber regeneration. (A) Collagen content in skeletal muscles of WT and KO mice, measured with a Sirius Red Collagen kit $(n=4-6)$.

(B) Representative Masson's trichrome staining for collagen in muscle sections of WT and KO mice. (C) Quantitative real-time PCR ( $\mathrm{q}-\mathrm{PCR}$ ) analysis of mRNA level of fibrosis regulator TCF- $\beta 1$ in WT and KO muscle ( $n=5-7)$. (D) q-PCR analysis of mRNA levels of collagen genes, as well as genes related to fibrosis such as vimentin (Vim) and fibronectin 1 (Fn1), in WT and KO muscle $(n=5-7)$. (E) Representative $\mathrm{H} \& \mathrm{E}$ staining on tibialis anterior muscle cryosections of WT and KO mice.

Tissues were isolated at days $0,3,7$, and 14 after cardiotoxin (CTX) injury. Arrows indicate infiltrated adipocytes. The bottom panels show representative Oil Red O (ORO) staining on muscle cryosections of WT and KO mice at 14 days after injury. Scale bars: $50 \mu \mathrm{m}$. The experiment was replicated 2 times with 3 or 4 mice per group. Data are represented as mean \pm SEM. Two-tailed Student's $t$ test ( $\mathbf{A}$ and $\mathbf{C}$ ) or Bonferroni's multiple comparison test (D) were used to determine the statistical significance. ${ }^{*} P<0.05 ;{ }^{* *} P<0.001$.

rounds of degeneration and regeneration, resulting in a diminished reparative capacity accompanied by fibroblast proliferation and deposition of collagen and other extracellular matrix proteins (38, 39). To determine if cavin-1 null skeletal muscle is fibrotic, we measured total collagen and observed a roughly 2 -fold increase in these proteins (Figure 4A). As shown in Figure 4B, Masson's trichrome staining confirmed that collagen

levels were elevated in the muscle fibers of cavin-1 null mice. In accordance, the mRNA expression of the fibrosis regulator TGF- $\beta$ was also markedly increased with cavin-1 deletion (Figure 4C). Lastly, we analyzed the gene expression of various collagens as well as vimentin ( Vim) and fibronectin (Fn1), which are fibrosis-related genes. As determined by q-PCR, the mRNA levels of collagen 6 isoforms in particular were significantly elevated in cavin-1 null muscles (Figure 4D) as were those for fibronectin and vimentin.

We observed a marked number of muscle fibers with centrally located nuclei in cavin-1 null mice (Figure 1, C and E), suggesting that cavin-1 is involved in skeletal muscle regeneration. To confirm this, we induced acute muscle injury and regeneration in mice by injecting cardiotoxin (CTX) into the TA muscle. As shown in Figure 4E, within 3 days after CTX injury, both WT and KO mice exhibited extensive myofiber degeneration, indicated by the necrosis of myofibers and the loss of muscle architecture. By day 7 following CTX injury, most injured myofibers were cleared and replaced by regenerated myofibers with centralized nuclei in both WT and KO mice, although there were more adipocytes present in the latter. On day 14 following CTX injection, muscle architecture was largely restored in both 
A

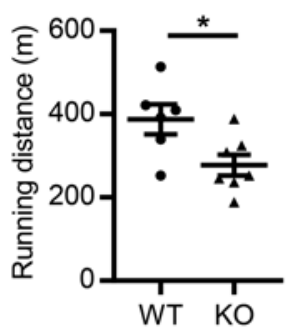

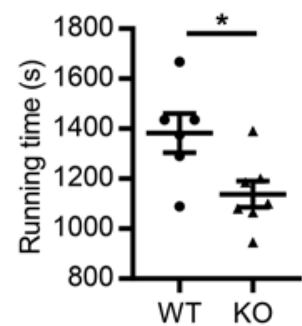

B

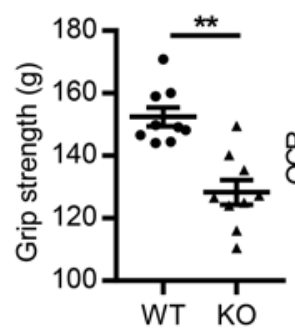

C

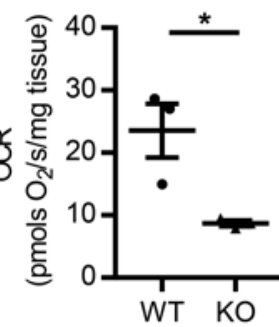

D

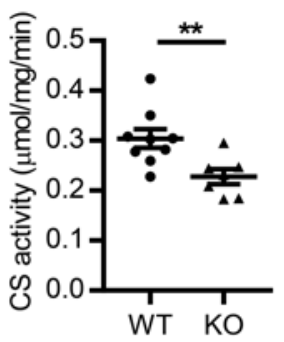

E
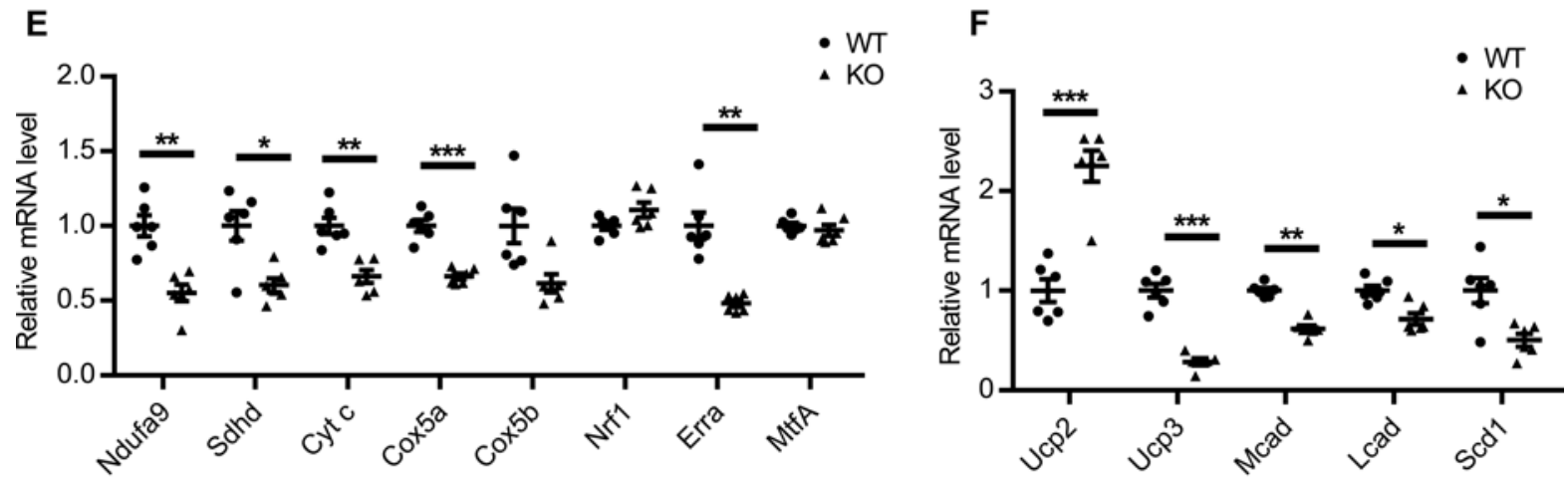

G

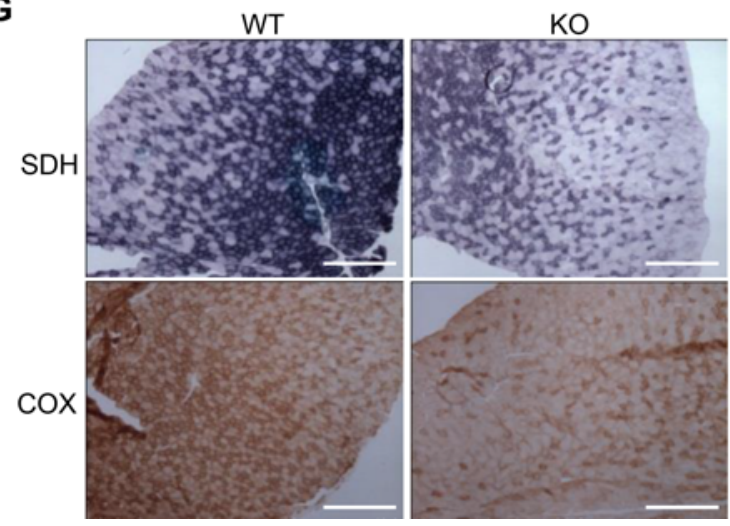

H

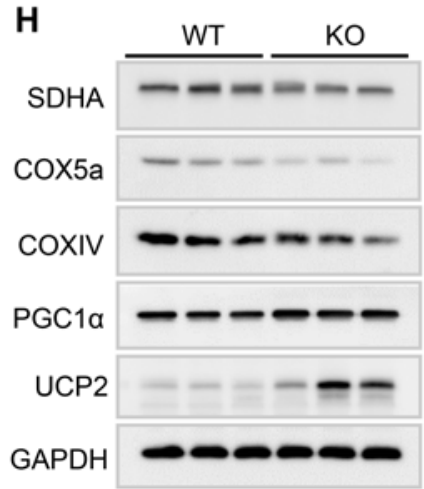

I

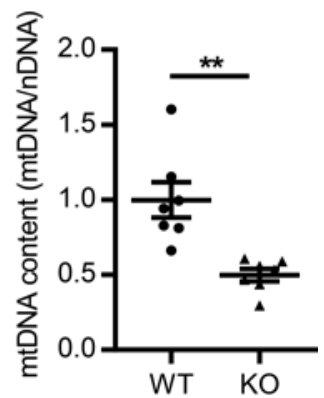

Figure 5. Deficiency of cavin-1 results in decreases in exercise tolerance, oxidative capacity, and the expression of mitochondrial and transcriptional regulatory mitochondrial genes in muscles. (A) Treadmill exercises in 10-week-old WT and cavin-1 KO mice, showing significantly decreased running distance and running time in KO mice $(n=6-7)$. (B) Forelimb grip strength test in 10-week-old WT and cavin-1 KO mice $(n=9)$. (C) Oxygen consumption rate (OCR) measured from soleus muscle fiber bundles isolated from WT and KO mice ( 3 mice/group). The substrates used were 5 mM malate plus 5 mM pyruvate. OCR associated with ATP synthesis rates was induced with $1 \mathrm{mM} \mathrm{ADP}$. (D) Citrate synthase (CS) activity in soleus muscle of WT and cavin-1 KO mice ( $n=$ 7-9). (E) Relative mRNA levels of genes involved in oxidative energy production in soleus muscles of WT and cavin-1 KO mice $(n=6)$. (F) Relative mRNA levels of genes involved in fatty acid oxidation in soleus muscles of WT and cavin-1 KO mice $(n=6)$. (C) Representative succinate dehydrogenase (SDH) and cytochrome oxidase (COX) histochemical staining on muscle cryosections from 12-week-old WT and cavin-1 KO mice. Scale bars: $500 \mu \mathrm{m}$. (H) Western blot analysis of proteins involved in respiratory chain complexes in muscles from 12-week-old WT and KO mice. (I) Mitochondrial DNA (mtDNA) copy number determined relative to nuclear DNA (nDNA) for soleus muscle DNA from WT and KO mice by quantitative real-time PCR analysis using primers for Nd1 (mitochondrial gene) and Tfam (nuclear gene) $(n=7)$. Data are represented as mean \pm SEM. Two-tailed Student's $t$ test (A-D and I) or Bonferroni's multiple comparison test ( $\mathbf{E}$ and $\mathbf{F}$ ) were used to determine the statistical significance. ${ }^{*} P<0.05 ;{ }^{* *} P<0.01 ;{ }^{* *} P<0.001$.

genotypes, suggesting that the absence of cavin-1 does not completely prevent muscle regeneration per se in the short term. However, fatty infiltration was clearly shown in cavin-1 null mice, as confirmed by the marked Oil Red O staining (Figure 4E), indicating impaired regeneration following acute injury, which is associated with disruption of muscle integrity and function (38).

Cavin-1 null mice have reduced muscle exercise capacity and exhibit defective skeletal muscle mitochondrial respiratory activity. We exercised mice and also measured their grip capacity to determine if the ablation of cavin-1 led to changes in muscle performance, as has been observed in humans lacking functional cavin-1 protein $(5,7,8,17)$. We subjected mice to treadmill running, and found that cavin-1 null mice indeed ran shorter 


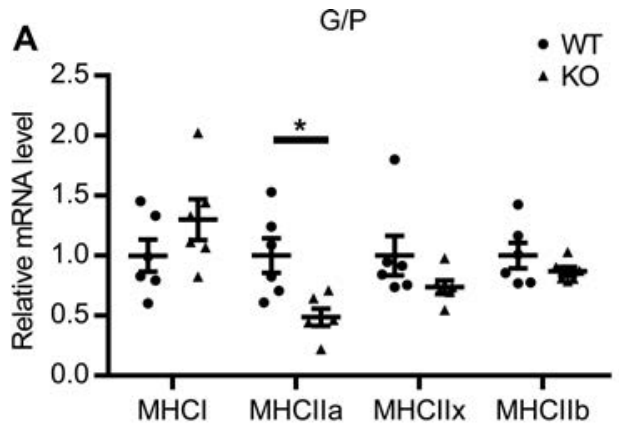

C
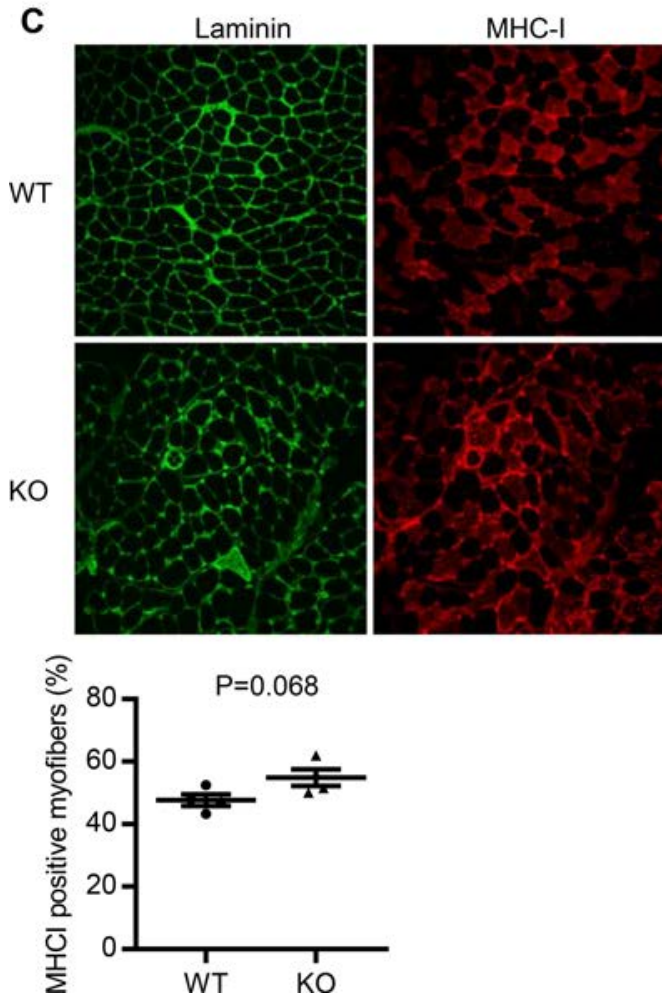

B

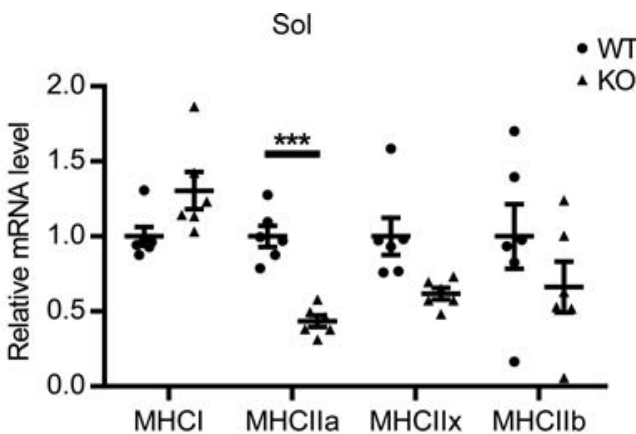

D
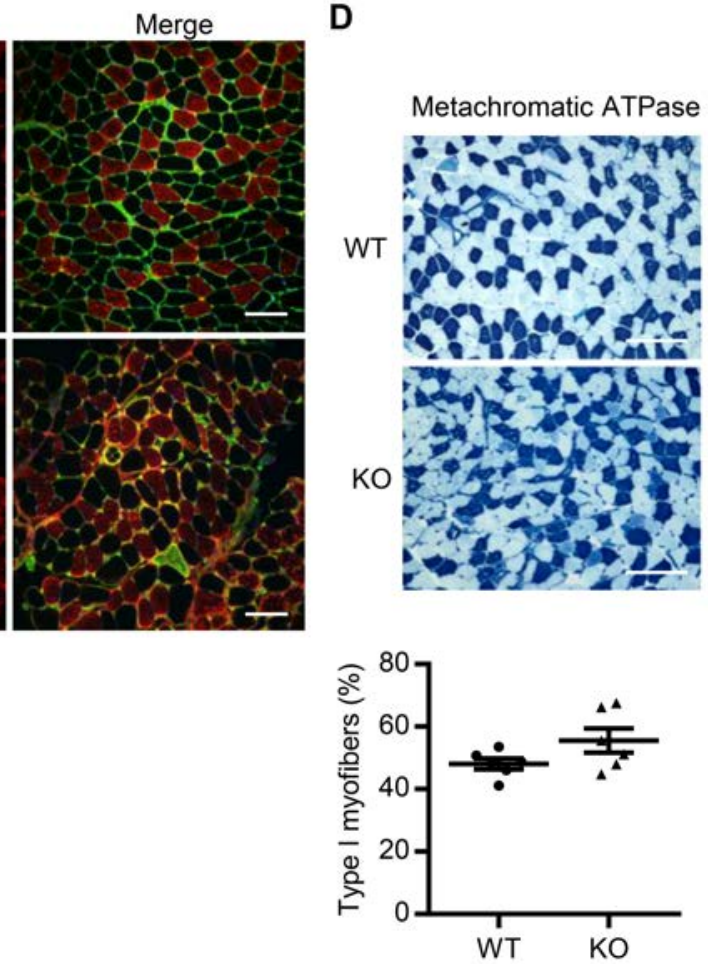

Figure 6. Cavin-1 KO mice exhibit alteration of skeletal muscle fiber type. (A) mRNA expression of myosin heavy chain (MHC) isoforms in gastrocnemius/plantaris (G/P) muscle, determined by quantitative real-time PCR $(n=6)$. (B) mRNA expression of MHC isoforms in soleus muscle $(n=6)$. (C) Representative immunofluorescence histochemistry using NOQ7.5.4D anti-MHC-I antibody on soleus muscle cryosections of WT and cavin-1 KO mice. Nuclei are stained with DAPI. Scale bars: $100 \mu \mathrm{m}$. Quantitative analysis of percentage of MHC-I-positive fibers (red) is shown in the bottom panel $(n=4)$, by using Image) software to count the number of total and positively stained myofibers. (D) Representative metachromatic ATPase staining on soleus muscle cryosections and quantitative analysis of type I fiber (dark blue) percentage $(n=6)$. Scale bars: $50 \mu \mathrm{m}$. Data are represented as mean $\pm \mathrm{SEM}$. Bonferroni's multiple comparison test (A and B) or 2-tailed Student's $t$ test (C and $\mathbf{D}$ ) were used to determine the statistical significance. ${ }^{*} P<0.05 ;{ }^{* *} P$ $<0.001$.

distances ( $277 \mathrm{~m}$ vs. $388 \mathrm{~m}$ ) and for less time (1,138 seconds vs. 1,383 seconds) than WT mice, indicative of muscle abnormalities (Figure $5 \mathrm{~A}$ ). A forelimb grip strength test showed mild but statistically significant decreases for this parameter in cavin-1 null mice (Figure 5B). Treadmill exercise requires aerobic capacity, and we therefore measured the oxygen consumption rate (OCR) in isolated soleus muscle fibers from WT and KO mice using an Oxygraph system (Hansatech Instruments). The absence of cavin-1 resulted in significant declines in ADP-stimulated state 3 respiration (Figure 5C). The enzyme citrate synthase (CS) is involved in the first step of the TCA cycle and its activity is commonly used as a quantitative enzyme marker for the mitochondria intactness and aerobic capacity (40). CS activity in muscles of WT and KO mice was analyzed, and as shown in Figure 5D, CS activity in cavin-1 KO mice was reduced by $25 \%$ as compared with WT mice, consistent with a reduced mitochondrial content.

We investigated by q-PCR the possibility that changes in mitochondrial function in cavin-1 null mice could be due to changes in gene expression levels for those genes involved in oxidative phosphorylation. The data in Figure 5E show that many of these genes were indeed downregulated, including those encoding proteins involved in the different mitochondrial respiratory chain complexes (Ndufa9 in complex I, Sdhd in complex II, Cytc in complex III, and Cox $5 \mathrm{a}$ in complex IV). Except for genes encoding estrogen-related receptor $\alpha(E R R \alpha)$, the expression of mitochondrial regulatory genes, mitochondrial transcription factor A $(M t f A)$, and nuclear respiratory factor $1(N v f 1)$ was unchanged in $\mathrm{KO}$ mice. The genes related to fatty acid oxidation, such as $U c p 3, M c a d, L c a d$, and $S c d 1$ were significantly suppressed, whereas interestingly, the 
expression of $U c p 2$ was dramatically increased (Figure $5 \mathrm{~F}$ ). We then performed immunostaining of muscles for mitochondrial enzymes in the gastrocnemius muscle. The intensities of both succinate dehydrogenase $(\mathrm{SDH})$ and cytochrome oxidase (COX) staining were attenuated in cavin-1-deficient muscles (Figure 5G), further indicating decreased mitochondrial energetic activity. We confirmed reduced protein levels for mitochondrial protein expression by Western blot as shown in Figure $5 \mathrm{H}$, and these also occurred in different respiratory chain complexes: SDHA in complex II, as well as Cox5a and CoxIV in complex IV. Consistent with q-PCR results, Western blots also showed marked increase in UCP2 protein levels (Figure 5G). The increased content of UCP2 has been reported to be associated with reduced skeletal muscle lipid utilization (41), and we confirmed the mRNA data with protein expression. Interestingly, we also found a slight increase in peroxisome proliferator-activated receptor gamma coactivator $1 \alpha(\mathrm{PGC} 1 \alpha)$ protein levels in $\mathrm{KO}$ muscle, though the mRNA levels remained unchanged (data not shown), which could be explained as a compensatory effect in response to decreased oxidative capacity. We also evaluated mitochondrial DNA (mtDNA) copy number by q-PCR with primers for $N d 1$ (mitochondrial gene) and Tfam (nuclear gene). The data show significantly decreased mtDNA, which is consistent with the decreased CS activity, indicating decreased mitochondria number (Figure 5I).

Cavin-1 null mice have altered skeletal muscle fiber types. Skeletal muscle is composed of a spectrum of different fibers which range from slow-twitch oxidative type I, fast-twitch oxidative type IIa to fast-twitch glycolytic type IIb and type IIx, and the expression of myosin heavy chain (MHC) isoforms is used to classify fiber types (42). In general, the slow-twitch type I fibers predominantly rely on oxidative metabolism and contain a high mitochondrial content, compared with fast-twitch type II fibers. To examine whether the absence of cavin-1 alters muscle fiber-type composition, we analyzed $\mathrm{MHC}$ isoforms in various muscles from 12-week-old mice. q-PCR analysis revealed that in G/P muscles (Figure 6A) and soleus muscles (Figure 6B), type IIa MHC (MHC-IIa) significantly decreased, the levels of MHC-IIx and MHC-IIb remained unchanged, while type I MHC (MHC-I) showed an increasing trend, suggesting that loss of cavin-1 caused alteration in fiber-type composition, which could affect the muscle contractile function. A similar pattern of mRNA expression was also observed in TA muscles (data not shown). We also analyzed muscle fibertype composition from adult mice by immunostaining against MHC-I (Figure 6C) and by metachromatic ATPase staining (Figure 6D). Similar to the MHC-I mRNA results, there was no statistically significant difference between WT and KO muscle, although we observed a slightly increasing trend in the percentage of type I fibers in cavin-1-deficient muscle.

\section{Discussion}

The biochemical properties of PTRF/cavin-1 null skeletal muscle shown herein describe a unique form of $\mathrm{MD}$ in the general class of MDs caused by sarcolemmal protein defects (2). PTRF/cavin-1 in the context of caveolae was first described as an abundant peripheral membrane protein called cav-p60 that is highly expressed in fat and muscle caveolae - the sarcolemma in the latter case (43). It was later shown to be identical to PTRF (44) and to be essential for the formation of caveolae $(22,45,46)$. Ptrf null mice lack caveolae in all tissues examined, including skeletal muscle, and are lipodystrophic with a moderate insulin-resistant phenotype $(21,22)$. A number of studies have appeared describing humans lacking functional caveolae due to inactivating mutations in the PTRF gene (13). These patients, numbering about 30 and present in several families worldwide, were described as having a primary phenotype of CGL4, but all have myopathy/ $\mathrm{MD}$ of varying severity consistent with the general picture of the CGL phenotype. Other than a description of the patients and some muscle histology, little information is available concerning the underlying mechanism(s) that lead to the phenotype, the exception being that muscle hypertrophy could be a result of constitutive Akt activation seen in skeletal muscles from certain patients (5). Activation of the PI3K/Akt signaling pathway is a key regulator of skeletal muscle hypertrophy and mediates hypertrophic response in both dystrophin- and sarcoglycan-deficient mice, which are models for DMD and LGMD, respectively (47). IGF-1 induces Akt activation and is an upstream stimulator of muscle growth (27). Here we show that Akt in PTRF/cavin-1 null skeletal muscle is in fact constitutively activated in the KO mice, comparable to levels seen with IGF-1 stimulation in WT mice (Figure 2). As in humans, most muscles of the PTRF/ cavin-1 null mice are hypertrophic and multinucleated due in part to Akt activation.

Caveolae play a role in organizing cell surface membranes and in protecting them from mechanical and osmotic stress, amongst other proposed biochemical roles $(9,11)$. It is therefore not surprising that their lack in cavin-1 null mice disrupts the skeletal muscle sarcolemma, similar to what occurs in 
the most common types of $\mathrm{MD}$ that are due to deficiencies/mutations in dystrophin and other DGC components (48). The resultant muscle is mechanically fragile, and this damage provokes a regenerative response of muscle satellite cells that eventually becomes a chronic inflammatory response and causes pathology including fibrosis (Figures 3 and 4). The overall phenotype we observed fits this pattern of multinucleated, mostly hypertrophic muscle fibers that are fibrotic, but other biochemical features appear unique to the PTRF null phenotype.

The DGC forms a link between the intracellular cytoskeleton and the extracellular matrix and maintains sarcolemma mechanical stability. Loss of any of the protein components of the DGC complex in MD leads to loss of the other components and causes sarcolemmal instability and initiates downstream pathological processes and progressive muscle dysfunction $(2,30,31)$. On the other hand, we see an upregulation of the DGC components in the cavin-1 null mice (Figure 3), but the cause of MD and dysfunction is likely the same as in DGC deficiency, namely, loss of membrane stability. Consistent with this interpretation, there is an increased expression of proteins involved in membrane repair such as dysferlin and MG53, which have been postulated to facilitate vesicle translocation to the sites of membrane injury, and are involved in assembling a repair complex made up of caveolin-3 and PTRF/cavin-1 (34, 35, 49, 50). In common with other MDs, there are increased levels of circulating CK due to its leakage from myofibers. In addition to the dramatic, apparently compensatory increase in the expression of DGC components, we observed enhanced expression of cytoskeletal proteins, tubulin and vimentin, also an apparent compensation for lack of or compromised cytoskeletal-extracellular matrix linkage. These data are all consistent with loss of caveolae leading to an altered sarcolemmal organization and decreases in skeletal muscle function as assessed directly by aerobic exercise and strength determination.

Given the pleiotropic nature of the PTRF/cavin-1 null phenotype - muscle hypertrophy, membrane damage, and fibrosis - the functional deficiencies of strength and endurance are rather mild but in line with those of the dystrophin-deficient mouse $(48,51)$. A compensatory increase in muscle respiration might be expected, as a further compensation to maintain muscle function as has been proposed to account for the loss of calcium in MD. This is not the case, however, as cavin-1 null mice have a reduced respiratory capacity in their muscle and show deficiencies at the gene-expression level in numerous protein components of the mitochondrial respiratory chain and a significantly reduced number of mitochondria (Figure 5). In addition, histochemical staining for $\mathrm{SDH}$ and $\mathrm{COX}$ activities indicated that oxidative capacity is decreased in myofibers of $\mathrm{KO}$ mice. On the other hand, the enhanced muscle regeneration and increased fiber size in the $\mathrm{KO}$ mice might at least partially explain their relatively mild functional defects.

Cavin-1 null muscles display alterations in myofibers with distinctive contractile and metabolic properties. The q-PCR data suggested that the absence of cavin-1 significantly suppressed the type IIa myofiber genes, whereas there was an increasing trend in type I fiber genes. ATPase staining and fluorescence immunohistochemistry against MHC-I also suggested a slight increase in type I fibers. However, this tendency of fast-to-slow myofiber shift seems incompatible with the mitochondrial dysfunction implicated in cavin-1-deficient muscle, since type I fibers are believed to have higher oxidative enzyme activity and be more resistant to disease or damage than type II (fast-twitch) fibers (42). Although the exact mechanism underling this discrepancy and whether or not the fiber-type changes in $\mathrm{KO}$ muscle are secondary to the general phenotype are unclear, there are several possible explanations. One possibility is that the decrease in type IIa myofiber contributed the most to the decreased oxidative capacity in $\mathrm{KO}$ muscle, rather than type I fiber. It has been reported that the soleus muscles of mice had predominantly fast-oxidative type IIa fibers, followed by the slow-oxidative type I fibers, and very low percentages of glycolytic IIx/IIb types (52). Moreover, IIa and many IIx fibers could have a higher SDH activity than type I fibers in mouse and rat muscle (53). Thus, the abnormal oxidative capacity in a mouse model could be due to a significant decrease in type IIa fibers, rather than changes in type I fibers. Nevertheless, a slight increase in type I fibers could be a compensatory protective mechanism against the muscle weakness caused by mitochondrial dysfunction. Similar fiber-type switching toward type I has also been observed in DMD (54).

An interesting contrast exists between cavin-1 and Cav3 null mice, both of which lack muscle caveolae and have MD. In the latter model, the expression of DGC components is essentially unchanged, although the complex is excluded from lipid raft domains and the T-tubule network is disorganized (14). Also, mice with Cav3 deficiency exhibit muscle atrophy, which was rescued by myostatin inhibition $(55,56)$. In contrast, our data show that most skeletal muscle fibers were hypertrophic in mice lacking cavin-1. Muscle hypertrophy is also characteristic of human PTRF/cavin-1 deficiency $(5,8)$. In addition, 
hypertrophic cardiomyopathy has been reported in human CAV3 mutations (57) and in Cav3 null mice (58), as well as in cavin-1 null mice (59). It should be noted, however, that while PTRF mutations in mice and humans result in total loss of protein, most (or all) human CAV3 mutations encode altered proteins that are expressed and can act in a dominant-negative fashion, causing alterations/loss of caveolar structures. Thus, the phenotypes of mice and humans with CAV3 mutations cannot be directly compared with each other or with PTRF/cavin-1 loss. The latter causes a universal loss of caveolae, whereas the former only affects muscle.

Therefore, there are more secondary consequences for the $P T R F /$ cavin-1 null genotype in other tissues as compared with the CAV3 mutations, which lead to a particular and uniquely complex form of MD. The lipodystrophy caused by caveolae loss in fat cells due to PTRF/cavin-1 loss was already noted (21), and this results in an altered pattern of adipokine production. Adipokines affect other tissues in various ways including skeletal muscle where adiponectin, for example, enhances insulin sensitivity (60). There is also a further potential effect of PTRF/cavin-1 deficiency, namely that owing to its caveolae-independent function in the regulation of ribosomal RNA levels that has been demonstrated in adipocytes, which are terminally differentiated cells that require efficient ribosomal function for adequate cell nutrition (61). Whether or not this regulation applies to skeletal muscle is in the process of being experimentally addressed. To this end, generating muscle-specific PTRF/cavin-1 KO mice would also be beneficial for determining the physiological role of cavin-1 in postnatal skeletal muscle. In conclusion, the similarities in skeletal muscle abnormalities in KO mice and in human patients indicate that cavin-1 plays a critical role in maintaining normal muscle shape and function. The cavin-1 KO mice could be used as a powerful model to explore the potential involvement of caveolae in human muscle disease, to elucidate mechanisms underlying MD, and to provide promising therapeutic insights for CGL.

\section{Methods}

Animals. The cavin-1 KO mice were created as described on a C57BL/6N $\times$ sv129 genetic background and backcrossed for at least 8 generations with the C57BL/6N lineage $(21,22)$. All animals were generated from breeding of cavin-1 heterozygous mice. The animals were maintained in a pathogen-free animal facility at $21^{\circ} \mathrm{C}$ under a 12 -hour light/12-hour dark cycle with access to a standard rodent diet. The mice were food deprived but provided with water for 4 hours prior to experimental procedures. For tissue harvesting, mice were sacrificed under $\mathrm{CO}_{2}$ anesthesia, and tissues were rapidly taken and immediately frozen in liquid nitrogen and stored at $-80^{\circ} \mathrm{C}$ until biochemical analysis, or they were immediately fixed for histology and immunohistochemistry.

Muscle histological and immunofluorescence analyses. Muscle tissues from WT and cavin-1 null mice were isolated, and fixed with Z-Fix (Anatech Ltd.), embedded in paraffin, sectioned, and stained with H\&E or Masson's trichrome, or quickly frozen in isopentane cooled in liquid nitrogen. Sections ( $8 \mu \mathrm{m}$ thick) were obtained from the middle portion of frozen muscle and processed for histological and immunofluorescence analysis. Oil Red $\mathrm{O}$ staining and SDH and COX staining were performed at the Tufts Animal Histology Core (Tufts University, Boston, Massachusetts, USA). Metachromatic ATPase staining was performed as described previously (62). The fiber CSA and the number of centrally nucleated fibers were determined for H\&E-stained sections from 4 animals/genotype (> 800 cells/genotype) using ImageJ software (NIH).

For immunofluorescence histochemical analysis, frozen sections were fixed in $4 \%$ paraformaldehyde, permeabilized with $0.1 \%$ Triton X-100/PBS, and incubated with specific primary antibodies followed by secondary antibodies, then detected with immunofluorescence microscopy (Nikon deconvolution wide-field epifluorescence system). The following primary and secondary antibodies were used at the indicated dilutions: laminin (Sigma-Aldrich, catalog L9393, 1:30), $\beta$-dystroglycan (Leica Biosystems, catalog NCL-b-DG, 1:100), dystrophin (Leica Biosystems, catalog NCL-Dys, 1:50), MHC-I (Sigma-Aldrich, clone NOQ7.5.4D, 1:100), Alexa Fluro 594-conjugated goat anti-mouse IgG (Invitrogen, catalog A11032, 1:200), and Alexa Fluro 488-conjugated goat anti-rabbit IgG (Invitrogen, catalog A11034, 1:200). The fluorescence intensity was analyzed using ImageJ software.

CTX injury. CTX (50 $\mu 1$ of $10 \mu \mathrm{M})$ from Naja mossambica mossambica (Sigma-Aldrich) was injected into the TA muscle under general anesthesia. As a control, $0.9 \%$ saline solution was injected into the contralateral extremity. For muscle regeneration analyses, mice were sacrificed at days 3, 7, and 14 after injection, and TA muscle was excised and frozen in isopentane cooled to $-165^{\circ} \mathrm{C}$ in liquid nitrogen. $\mathrm{H} \& \mathrm{E}$ staining and Oil Red $\mathrm{O}$ staining were performed on cryosections. 
Treadmill analysis. Mice were assimilated for treadmill running in a chamber interfaced with gas analyzers (Oxymax, Columbus Instruments) for 3 consecutive days. Daily exercise duration was 10 minutes, which progressed from a stationary position at a $10^{\circ}$ incline to a speed of $10 \mathrm{~m} / \mathrm{min}$, allowing the mice to assimilate to handling and running on the treadmill lane. After 3 days of assimilation, the exercise tolerance of the mice was determined by a stepwise progressive test, during which the mice initially ran at a speed of $10 \mathrm{~m} / \mathrm{min}$ and a $10^{\circ}$ incline for 3 minutes. The speed of the treadmill was increased by $2 \mathrm{~m} / \mathrm{min}$ every 2 minutes until they were unable to avoid an electrical shock grid at the back of the treadmill for 5 consecutive seconds, at which time the electrical stimulus was turned off. The running time and running distance were recorded.

Forelimb grip strength test. An automated Grip Strength Meter (Columbus Instruments) was used to measure muscle strength of 10- to 12-week-old cavin-1 null and WT mice. Forelimb grip strength was automatically recorded in real time with a digital force-gauging apparatus. Seven readings achieved by each mouse were recorded and with the highest and lowest number excluded, and the average of the remaining 5 readings was considered as the final grip strength.

IGF-I treatment. Mice under anaesthetization were injected with $50 \mu \mathrm{lof} 50 \mu \mathrm{g} / \mathrm{ml}$ recombinant IGF-I protein (Sigma-Aldrich) into the TA muscles, and the contralateral TA muscle was injected with $50 \mu 1$ of saline. Both muscles were removed 15 minutes after the injections and immediately frozen in liquid nitrogen (63).

Assays. Collagen content in the muscles was determined using a Sirius Red Collagen Detection kit (Chondrex Inc.) according to the manufacturer's instructions. Briefly, $25 \mathrm{mg}$ of hindlimb muscle tissues from WT and cavin-1 KO mice was extracted in acetic acid, and then incubated with Sirius Red solution. The absorbance was read at $546 \mathrm{~nm}$. For CK, sera were collected and analyzed immediately without freezing. CK activity assays were performed with a commercial kit (Stanbio CK Liqui-UV Test, Stanbio Laboratory) according to the manufacturer's instruction. The absorbance was read at $340 \mathrm{~nm}$. Akt activity was determined with Akt Kinase Assay Kit (Cell Signaling Technology). Briefly, Akt was immunoprecipitated from $400 \mu \mathrm{g}$ of muscle lysates, and incubated with a GSK-peptide substrate. Kinase activity was measured using an anti-p-GSK3 $\alpha / \beta$ (Ser21/9) antibody (included in the kit) to detect phosphorylation by Western blot analysis.

CS activity was measured in soleus muscle homogenates from WT and cavin-1 KO mice as described previously with some modification (64). Muscle was homogenized on ice in $0.1 \mathrm{M}$ Tris buffer containing $0.1 \%$ Triton X-100. The homogenates were frozen under liquid nitrogen and thawed 4 times to disrupt the mitochondria. The assay system contained 0.1 M Tris- $\mathrm{HCl}(\mathrm{pH} 8.5), 0.1 \mathrm{mM}$ 5,5'-dithiobis(2-nitrobenzoate) (DTNB), $0.31 \mathrm{mM}$ acetyl-CoA, $0.5 \mathrm{mM}$ oxaloacetate (OAA), and $5 \mu$ of muscle homogenate in a total volume of $200 \mu \mathrm{l}$, and the reaction was initiated with OAA. The rate of DTNB reduction before the OAA addition was subtracted from the rate after OAA addition. The color change was monitored at a wavelength of $412 \mathrm{~nm}$ at $25^{\circ} \mathrm{C}$. The CS activity was normalized to the total protein content and is reported as nanomoles per milligram protein per minute.

High-resolution respirometry of permeabilized muscle fibers. Respiratory activity was assessed in soleus fiber bundles using an Oxygraph system (Hansatech Instruments) as described by De Palma et al. (65). Fibers from soleus muscle were prepared in ice-cold BIOPS (50 mM K+-MES, $20 \mathrm{mM}$ taurine, $0.5 \mathrm{mM}$ dithiothreitol, 6.56 $\mathrm{mM} \mathrm{MgCl}_{2}, 5.77 \mathrm{mM}$ ATP, $20 \mathrm{mM}$ imidazole, $10 \mathrm{mM} \mathrm{Ca-EGTA} \mathrm{buffer,} \mathrm{pH}$ 7.1), and then permeabilized in 2 $\mathrm{ml}$ of BIOPS solution containing $50 \mu \mathrm{g} / \mathrm{ml}$ saponin by gentle agitation for 30 minutes at $4^{\circ} \mathrm{C}$. After rinsing for 10 minutes in ice-cold mitochondrial respiration medium (MiR06; $110 \mathrm{mM}$ sucrose, $60 \mathrm{mM}$ K-lactobionate, $0.5 \mathrm{mM}$ EGTA, $3 \mathrm{mM} \mathrm{MgCl}_{2}, 20 \mathrm{mM}$ taurine, $10 \mathrm{mM} \mathrm{KH}_{2} \mathrm{PO}_{4}, 20 \mathrm{mM} \mathrm{HEPES}, 1 \mathrm{~g} / 1 \mathrm{BSA}$, and $280 \mathrm{U} / \mathrm{ml}$ catalase, $\mathrm{pH}$ 7.1), the muscle fibers were weighed and added to the Oxygraph respiratory chamber. Respiration was measured in MiR06 at $37^{\circ} \mathrm{C}$. With $1 \mathrm{mM}$ ADP, in the presence of $5 \mathrm{mM}$ malate and $5 \mathrm{mM}$ pyruvate, coupled respiration is maximally stimulated. Respiration rates are expressed as $\mathrm{pmol}_{2} / \mathrm{s} / \mathrm{mg}$ dried fiber.

Quantification of mtDNA content. DNA was extracted from soleus muscle using a DNeasy Blood \& Tissue Kit (Qiagen). mtDNA content was determined by q-PCR analysis. The levels of NADH dehydrogenase 1 (Nd1, mitochondrial gene) were normalized to the levels of transcription factor A, mitochondrial (Tfam, nuclear gene) to evaluate the mitochondria number. The primer sequences can be found in Supplemental Table 1.

$q-P C R$. Total RNA was isolated from indicated tissues with TRIzol reagent (Invitrogen) and the cDNA was synthesized using a Reverse Transcription System (Promega). q-PCR was performed with the ViiA7 detection system (Applied Biosystems) using Fast SYBR Green Master Mix (Applied Biosystems) according to the manufacturer's protocol. Gene expression levels were normalized to 36B4. The primer sequences can be found in Supplemental Table 1. 
Western blot analysis. Protein was extracted using RIPA lysis buffer and the concentration was determined using a Pierce BCA protein assay kit (Thermo Fisher Scientific). The tissue homogenates were run in SDSPAGE and proteins were transferred to PVDF membranes. After blocking with $10 \%$ nonfat dry milk in PBST, the membranes were incubated with primary antibodies and then horseradish peroxidase-conjugated secondary antibodies (Sigma-Aldrich). Chemiluminescent signals were developed with enhanced chemiluminescence reagents (PerkinElmer), followed by detection using a Fujifilm LAS-4000 Image Analyzer.

Antibodies against the following proteins were used: dysferlin (catalog NCL-Hamlet) and $\alpha$-sarcoglycan (catalog NCL- $\alpha$-SARC) from Leica Biosystems; SDHA (catalog 5839S), cytochrome $c$ (catalog 4272), GSK-3 $\alpha / \beta$ (catalog 5676), phospho-GSK-3 $\alpha / \beta$ (Ser21/9) (catalog 9327), 4E-BP1 (catalog 9644), phospho-4E-BP1 (Ser65) (catalog 9451), S6 ribosomal protein (catalog 2217), phospho-S6 ribosomal protein (Ser235/236) (catalog 2211), phospho-p70S6 kinase (Thr389) (catalog 9206), Akt (catalog 9272) and phospho-Akt (Ser473) (catalog 4060) from Cell Signaling Technology; Cox4 (catalog sc-58348), Cox5a (catalog sc-376907), UCP2 (catalog sc-390189), MyoD (catalog sc-760), PGC1 $\alpha$ (catalog sc-13067), vimentin (catalog sc-32322) and P70S6K (catalog sc-230) from Santa Cruz Biotechnology; TRIM72 (MG53; catalog PA5-19398) and RyR1 (catalog MA3-916) from Thermo Fisher Scientific; Cav1 (catalog 610059) and Cav-3 (catalog 610420) from BD Biosciences; DHPR $\alpha 2$ (catalog ab2864) from Abcam; and $\alpha$-actin (catalog GTX101362) from GeneTex.

Statistics. Data are presented as means \pm SEM. The significance of differences between groups was evaluated using 2-tailed unpaired Student's $t$ test or Bonferroni's multiple comparison test where appropriate. A $P$ value less than 0.05 was considered significant. The statistical analyses were carried out using Prism 7 (GraphPad Software).

Study approval. All animal studies were performed in accordance with the guidelines and under approval of the IACUC of Boston University.

\section{Author contributions}

SD designed research studies, conducted experiments, analyzed data, and wrote the manuscript. LL discussed the data, reviewed and edited the manuscript. PP supervised the work, discussed the data, wrote and edited the manuscript.

\section{Acknowledgments}

We thank Marcus Oliveira from the Department of Medicine, Boston University School of Medicine for the technical help with the Oxygraph experiment and Thomas Balon from Metabolic Phenotyping Core at the Boston University Medical Campus for the help with treadmill and grip strength tests. This work was supported by the grants from the NIH (DK-30425 and DK-092942) to PFP.

Address correspondence to: Paul F. Pilch, Department of Biochemistry, Boston University School of Medicine, 72 E. Concord Street, Boston, Massachusetts 02118. USA. Phone: 617.638.4044; E-mail: ppilch@bu.edu.

1. Guiraud S, Aartsma-Rus A, Vieira NM, Davies KE, van Ommen GJ, Kunkel LM. The pathogenesis and therapy of muscular dystrophies. Annu Rev Genomics Hum Genet. 2015;16:281-308.

2. Nigro V, Piluso G. Spectrum of muscular dystrophies associated with sarcolemmal-protein genetic defects. Biochim Biophys Acta. 2015;1852(4):585-593

3. Galbiati F, Razani B, Lisanti MP. Caveolae and caveolin-3 in muscular dystrophy. Trends Mol Med. 2001;7(10):435-441.

4. Gazzerro E, Bonetto A, Minetti C. Caveolinopathies: translational implications of caveolin-3 in skeletal and cardiac muscle disorders. Handb Clin Neurol. 2011;101:135-142.

5. Hayashi YK, et al. Human PTRF mutations cause secondary deficiency of caveolins resulting in muscular dystrophy with generalized lipodystrophy. J Clin Invest. 2009;119(9):2623-2633.

6. de Haan W. Lipodystrophy and muscular dystrophy caused by PTRF mutations. Clin Genet. 2010;77(5):436-437.

7. Shastry S, Delgado MR, Dirik E, Turkmen M, Agarwal AK, Garg A. Congenital generalized lipodystrophy, type 4 (CGL4) associated with myopathy due to novel PTRF mutations. Am J Med Genet A. 2010;152A(9):2245-2253.

8. Ardissone A, et al. Novel PTRF mutation in a child with mild myopathy and very mild congenital lipodystrophy. $B M C M e d$ Genet. 2013;14:89.

9. Bastiani M, Parton RG. Caveolae at a glance. J Cell Sci. 2010;123(Pt 22):3831-3836.

10. Hansen CG, Nichols BJ. Exploring the caves: cavins, caveolins and caveolae. Trends Cell Biol. 2010;20(4):177-186.

11. Parton RG, del Pozo MA. Caveolae as plasma membrane sensors, protectors and organizers. Nat Rev Mol Cell Biol. 2013;14(2):98-112.

12. Cheng JP, Nichols BJ. Caveolae: one function or many? Trends Cell Biol. 2016;26(3):177-189. 
13. Patni N, Garg A. Congenital generalized lipodystrophies--new insights into metabolic dysfunction. Nat Rev Endocrinol. 2015;11(9):522-534.

14. Galbiati F, et al. Caveolin-3 null mice show a loss of caveolae, changes in the microdomain distribution of the dystrophin-glycoprotein complex, and T-tubule abnormalities. J Biol Chem. 2001;276(24):21425-21433.

15. Hagiwara Y, et al. Caveolin-3 deficiency causes muscle degeneration in mice. Hum Mol Genet. 2000;9(20):3047-3054.

16. Woodman SE, Sotgia F, Galbiati F, Minetti C, Lisanti MP. Caveolinopathies: mutations in caveolin-3 cause four distinct autosomal dominant muscle diseases. Neurology. 2004;62(4):538-543.

17. Dwianingsih EK, et al. A Japanese child with asymptomatic elevation of serum creatine kinase shows PTRF-CAVIN mutation matching with congenital generalized lipodystrophy type 4. Mol Genet Metab. 2010;101(2-3):233-237.

18. Rajab A, et al. Fatal cardiac arrhythmia and long-QT syndrome in a new form of congenital generalized lipodystrophy with muscle rippling (CGL4) due to PTRF-CAVIN mutations. PLoS Genet. 2010;6(3):e1000874.

19. Murakami N, et al. Congenital generalized lipodystrophy type 4 with muscular dystrophy: clinical and pathological manifestations in early childhood. Neuromuscul Disord. 2013;23(5):441-444.

20. Jelani M, et al. Novel nonsense mutation in the PTRF gene underlies congenital generalized lipodystrophy in a consanguineous Saudi family. Eur J Med Genet. 2015;58(4):216-221.

21. Ding SY, Lee MJ, Summer R, Liu L, Fried SK, Pilch PF. Pleiotropic effects of cavin-1 deficiency on lipid metabolism. J Biol Chem. 2014;289(12):8473-8483.

22. Liu L, et al. Deletion of Cavin/PTRF causes global loss of caveolae, dyslipidemia, and glucose intolerance. Cell Metab. 2008;8(4):310-317.

23. Lo HP, et al. The caveolin-cavin system plays a conserved and critical role in mechanoprotection of skeletal muscle. J Cell Biol. 2015;210(5):833-849.

24. Tapscott SJ. The circuitry of a master switch: Myod and the regulation of skeletal muscle gene transcription. Development. 2005;132(12):2685-2695.

25. Glass DJ. Molecular mechanisms modulating muscle mass. Trends Mol Med. 2003;9(8):344-350.

26. Glass DJ. Skeletal muscle hypertrophy and atrophy signaling pathways. Int J Biochem Cell Biol. 2005;37(10):1974-1984

27. Rommel C, et al. Mediation of IGF-1-induced skeletal myotube hypertrophy by PI(3)K/Akt/mTOR and PI(3)K/Akt/GSK3 pathways. Nat Cell Biol. 2001;3(11):1009-1013.

28. Schiaffino S, Dyar KA, Ciciliot S, Blaauw B, Sandri M. Mechanisms regulating skeletal muscle growth and atrophy. FEBS J. 2013;280(17):4294-4314.

29. Bodine SC, Baehr LM. Skeletal muscle atrophy and the E3 ubiquitin ligases MuRF1 and MAFbx/atrogin-1. Am J Physiol Endocrinol Metab. 2014;307(6):E469-E484.

30. Matsumura K, Campbell KP. Dystrophin-glycoprotein complex: its role in the molecular pathogenesis of muscular dystrophies. Muscle Nerve. 1994;17(1):2-15.

31. Straub V, Campbell KP. Muscular dystrophies and the dystrophin-glycoprotein complex. Curr Opin Neurol. 1997;10(2):168-175.

32. Ohlendieck K, Campbell KP. Dystrophin-associated proteins are greatly reduced in skeletal muscle from mdx mice. J Cell Biol. 1991;115(6):1685-1694.

33. Duclos F, et al. Progressive muscular dystrophy in alpha-sarcoglycan-deficient mice. J Cell Biol. 1998;142(6):1461-1471.

34. Weisleder N, Takeshima H, Ma J. Mitsugumin 53 (MG53) facilitates vesicle trafficking in striated muscle to contribute to cell membrane repair. Commun Integr Biol. 2009;2(3):225-226.

35. Bansal D, Campbell KP. Dysferlin and the plasma membrane repair in muscular dystrophy. Trends Cell Biol. 2004;14(4):206-213

36. Ozawa E, Hagiwara Y, Yoshida M. Creatine kinase, cell membrane and Duchenne muscular dystrophy. Mol Cell Biochem. 1999;190(1-2):143-151.

37. Govender P, et al. Cavin1; a regulator of lung function and macrophage phenotype. PLoS ONE. 2013;8(4):e62045

38. Mann CJ, et al. Aberrant repair and fibrosis development in skeletal muscle. Skelet Muscle. 2011;1(1):21.

39. Porter JD, et al. A chronic inflammatory response dominates the skeletal muscle molecular signature in dystrophin-deficient mdx mice. Hum Mol Genet. 2002;11(3):263-272.

40. Blomstrand E, Rådegran G, Saltin B. Maximum rate of oxygen uptake by human skeletal muscle in relation to maximal activities of enzymes in the Krebs cycle. J Physiol (Lond). 1997;501(Pt 2):455-460.

41. Simoneau JA, Kelley DE, Neverova M, Warden CH. Overexpression of muscle uncoupling protein 2 content in human obesity associates with reduced skeletal muscle lipid utilization. FASEB J. 1998;12(15):1739-1745.

42. Schiaffino S, Reggiani C. Fiber types in mammalian skeletal muscles. Physiol Rev. 2011;91(4):1447-1531.

43. Voldstedlund M, Vinten J, Tranum-Jensen J. cav-p60 expression in rat muscle tissues. Distribution of caveolar proteins. Cell Tissue Res. 2001;306(2):265-276.

44. Vinten J, Johnsen AH, Roepstorff P, Harpøth J, Tranum-Jensen J. Identification of a major protein on the cytosolic face of caveolae. Biochim Biophys Acta. 2005;1717(1):34-40.

45. Liu L, Pilch PF. A critical role of cavin (polymerase I and transcript release factor) in caveolae formation and organization. J Biol Chem. 2008;283(7):4314-4322.

46. Hill MM, et al. PTRF-Cavin, a conserved cytoplasmic protein required for caveola formation and function. Cell. 2008;132(1):113-124

47. Peter AK, Crosbie RH. Hypertrophic response of Duchenne and limb-girdle muscular dystrophies is associated with activation of Akt pathway. Exp Cell Res. 2006;312(13):2580-2591.

48. Durbeej M, Campbell KP. Muscular dystrophies involving the dystrophin-glycoprotein complex: an overview of current mouse models. Curr Opin Genet Dev. 2002;12(3):349-361.

49. Cai C, et al. Membrane repair defects in muscular dystrophy are linked to altered interaction between MG53, caveolin-3, and dysferlin. J Biol Chem. 2009;284(23):15894-15902.

50. Zhu H, et al. Polymerase transcriptase release factor (PTRF) anchors MG53 protein to cell injury site for initiation of membrane repair. J Biol Chem. 2011;286(15):12820-12824.

51. Watchko JF, O'Day TL, Hoffman EP. Functional characteristics of dystrophic skeletal muscle: insights from animal models J Appl Physiol. 2002;93(2):407-417. 
52. Gali Ramamoorthy T, et al. The transcriptional coregulator PGC-1 $\beta$ controls mitochondrial function and anti-oxidant defence in skeletal muscles. Nat Commun. 2015;6:10210.

53. Schiaffino S. Fibre types in skeletal muscle: a personal account. Acta Physiol (Oxf). 2010;199(4):451-463

54. Wang JF, Forst J, Schröder S, Schröder JM. Correlation of muscle fiber type measurements with clinical and molecular genetic data in Duchenne muscular dystrophy. Neuromuscul Disord. 1999;9(3):150-158.

55. Ohsawa Y, et al. Muscular atrophy of caveolin-3-deficient mice is rescued by myostatin inhibition. J Clin Invest. 2006;116(11):2924-2934.

56. Sunada Y, et al. Transgenic mice expressing mutant caveolin-3 show severe myopathy associated with increased nNOS activity. Hum Mol Genet. 2001;10(3):173-178.

57. Hayashi T, et al. Identification and functional analysis of a caveolin-3 mutation associated with familial hypertrophic cardiomyopathy. Biochem Biophys Res Commun. 2004;313(1):178-184.

58. Woodman SE, et al. Caveolin-3 knock-out mice develop a progressive cardiomyopathy and show hyperactivation of the p42/44 MAPK cascade. J Biol Chem. 2002;277(41):38988-38997.

59. Taniguchi T, et al. PTRF/cavin-1 deficiency causes cardiac dysfunction accompanied by cardiomyocyte hypertrophy and cardiac fibrosis. PLoS ONE. 2016;11(9):e0162513.

60. Caselli C. Role of adiponectin system in insulin resistance. Mol Genet Metab. 2014;113(3):155-160.

61. Liu L, Pilch PF. PTRF/Cavin-1 promotes efficient ribosomal RNA transcription in response to metabolic challenges. Elife. $2016 ; 5$.

62. Naya FJ, Mercer B, Shelton J, Richardson JA, Williams RS, Olson EN. Stimulation of slow skeletal muscle fiber gene expression by calcineurin in vivo. J Biol Chem. 2000;275(7):4545-4548.

63. Spangenburg EE, Le Roith D, Ward CW, Bodine SC. A functional insulin-like growth factor receptor is not necessary for loadinduced skeletal muscle hypertrophy. J Physiol (Lond). 2008;586(1):283-291.

64. Siu PM, Donley DA, Bryner RW, Alway SE. Citrate synthase expression and enzyme activity after endurance training in cardiac and skeletal muscles. J Appl Physiol. 2003;94(2):555-560.

65. De Palma C, et al. Deficient nitric oxide signalling impairs skeletal muscle growth and performance: involvement of mitochondrial dysregulation. Skelet Muscle. 2014;4(1):22. 